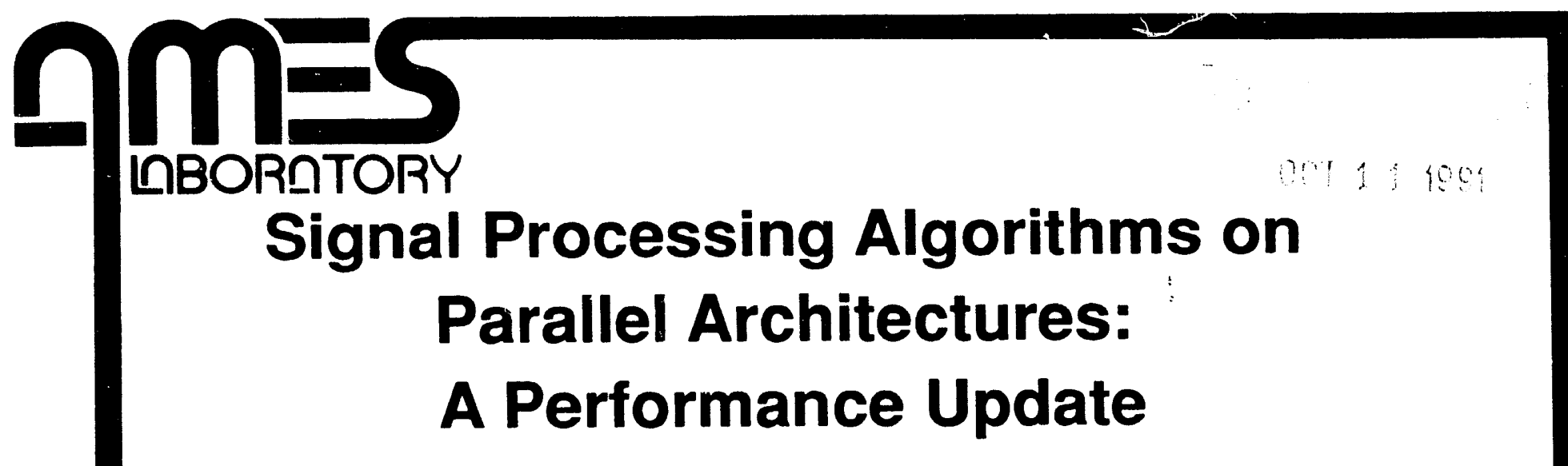

by

\author{
DIANE ROVER \\ VICKI TSAI \\ YIN-SHAN CHOW \\ JOHN GUSTAFSON
}

Ames Laboratory*

Iowa State University

Ames, IA 50011-3020

Date Transmitted: February, 1991

"Operated by lowa State University for the U.S. Department of Energy under contract No.

W-7405-ENG-82. 


\title{
Signal Processing Algorithms on Parallel Architectures: A Performance Update
}

\author{
by \\ DIANE ROVER \\ VICKI TSAI \\ YIN-SHAN CHOW \\ JOHN GUSTAFSON
}

Ames Laboratory*

Iowa State University

Ames, IA 50011-3020

Date Transmitted: February, 1991

* Operated by Iowa State University for the U.S. Department of Energy under contract No. W-7405-ENG-82. 


\section{DISCLAIMER}

This report was prepared as an account of work sponsored by an agency of the United States Government. Neither the United States Government nor any agency thereof, nor any of their employees, makes any warranty, express or implied, or assumes any legal liability or responsibility for the accuracy, completeness or usefulness of any information, apparatus, prociuct, or process disclosed, or represents that its use would not infringe privately owned rights. Reference herein to any specific commercial product, process, or service by trade name, trademark, manufacturer, or otherwise, does not necessarily constitute or imply its endorsement, recommendation, or favoring by the United States Government or any agency thereof. The views and opinions of authors expressed herein do not necessarily state or reflect those of the United States Government or any agency thereof.

This report has been reproduced directly from the best available copy.

AVAILABILITY:

To DOE and DOE contractors: Office of Scientific and Tgchnical Information P.O. Box 62

Oak Ridge, TN 37831

prices available from: (615) 576-8401

FTS: $626-8401$

To the public:

National Technical Information Service

U.S. Department of Commerce

5285 Port Royal Road

Springfield, VA 22161 


\section{TABLE OF CONTENTS}

Abstract 1

1. Introduction 1

2. Revisiting the Earlier Study 2

2.1. Serial Burg Algorithm 2

2.2. Summary of Performance Results 3

3. Performance Update 5

3.1. General Parallel Implementation 5

3.2. MasPar Implementation: A SIMD Update 5

3.3. nCUBE Implementation: A MIMD Update 9

4. Discussion 11

Acknowledgments $\quad 12$

References 13

Distribution List 15 


\title{
Signal Processing Algorithms on Parallel Architectures: A Performance Update*
}

\author{
DIANE ROVER ${ }^{\dagger}$, VICKI TSAI, YIN-SHAN CHOW, AND JOHN GUSTAFSON \\ Ames Laboratory, Iowa State University, Ames, IA 50011
}

\begin{abstract}
The Burg algorithm is a widely applied and extensively studied signal processing procedure having a structure typical of a class of important batch signal processing algorithms. Its implementation and performance on four different parallel machines were reported in the 1990 Journal of Parallel and Distributed Computing Special Issue on Massively Parallel Computation. The machines were: the Intel iPSC/2, the Denelcor HEP, the NASA/Goodyear MPP, and the Cray X-MP/48. The objective of the work reported here was to extend that study to two new parallel machines: the nCUBE 2 and the MasPar MP-1. These computers are related to the distributed memory systems above (i.e., the iPSC and the MPP, respectively), but use newer technology. In addition to achieving significant performance gains on the new machines compared to machines in the same architectural class, we found that the original study underestimated the scalability of the algorithm. That is, the algorithm maps efficiently to small-scale as well as large-scale computers, including both SIMD and MIMD distributed memory systems. Improvements in the parallel algorithm are highlighted. Of special import is the use of appropriate performance metrics and performance visualization to characterize the parallelism of the algorithm and lend insight toward understanding and evaluating its performance.
\end{abstract}

\section{INTRODUCTION}

The Burg algorithm [1] is a linear signal processing procedure for fitting an autoregressive model to a time series data set. It is a popular, widely applied, extensively studied, and computationally efficient method; its structure is typical of a large class of important batch signal processing algorithms (for example, in power spectrum estimation) [7]. To investigate the mapping of signal processing algorithms onto general purpose parallel computers, Sammur and Hagan [14] implemented the Burg algorithm on four different parallel machines: the Intel iPSC/2, the Denclcor HEP, the NASA/Goodyear MPP, and the Cray X-MP/48. Results of their cross-architectural study indicate that the algorithm has a high degree of parallelism. The objective of the work reported here was to extend that study to two new parallel machines recently acquired by the Scalable Computing Laboratory (SCL) at the Ames Laboratory: the nCUBE 2, a distributed memory hypercube MIMD (Multiple Instruction Multiple Data) machinc; and the MasPar MP-1, a distributed memory mesh SIMD (Single Instruction Multiple Data) machine. These computers are related to the distributed memory systems above (i.c., the iPSC and the MPP, respectively), but, most notably, use newer technology. The SCL currently has a 64-node nCUBE and a 8192-node MasPar; machines of varying sizes exist at sites outside of the SCL.

In addition to achieving significant performance gains on the new machines compared to their architectural counterparts, we found that the Sammur and Hagan study underestimated the scalability of the algorithm.

\footnotetext{
* This work is supported by the Applied Mathematical Sciences Program of the Ames Laboratory-U.S. Department of Energy under contract number W-7405-ENG-82.

† Present address: Department of Electrical Engineering, Michigan State University, East Lansing, MI 48824
} 
That is, although the earlier study concluded that the parallelism of the Burg algorithm can only be exploited if a fine-grained, massively parallel machine such as the MPP is used, the algorithm can map efficiently to smallscale as well as large-scale computers, including both SIMD and MIMD distributed memory systems. It is this quality that we informally refer to as scalable. Scalability is one consideration in parallel system design (among programmability, flexibility, cost-effectiveness, speed, etc.). There have been several attempts at defining "scalability" in a formal manner, e.g. [11]. For purposes of this paper, we require a relative definition: an algorithm-machine combination is more scalable than another if and only if it has less parallel overhead for all possible numbers of processors greater than one. We believe this property is possessed by all extant definitions of scalability. "Parallel overhead" can arise from load imbalance, communication costs, re' idant work, etc., and can be attributed to the machine or the algorithm design. The concept of granularity also commonly arises in this context, and formal definitions exist, e.g. $[2,6]$. Informally, a computer is said to be "fine grained" if its communication is fast relative to its computation speed. An algorithm is "fine grained" if it requires a large amount of communication relative to the computation. This potentially gives rise to opposite design goals: whereas we wish to design computers as fine grained as possible to increase their flexibility and generality, we wish to create algorithms as coarse grained as possible to attain good performance on a broad range of architectures.

The structure of the Burg algorithm, being representative of a class of algorithms, has already been mentioned (as a reason it was selected for the Sammur and Hagan study). Indeed, the structure of a machine, an architecture, a program, or a problem is closely tied to its scalability and hence its breadth of effectiveness. In the context of the Burg algorithm, this paper brings to light the performance impact of using more scalable machines and algorithms. Of special import is the use of appropriate performance metrics and performance visualization to characterize the parallelism of the algorithm and lend insight toward understanding and evaluating its performance.

Section 2 summarizes the Sammur and Hagan study and the relevances to this study. Section 3 presents our parallel implementations, giving both SIMD and MIMD updates and the respective performance analyses. Section 4 concludes with a discussion of the results.

\section{REVISITING THE EARLIER STUDY}

This section summarizes the Sammur and Hagan study, in particular it presents the serial algorithm, performance results from the parallel implementations, and points of contention that will be addressed in the next section.

\subsection{Serial Burg Algorithm}

The objective of the Burg algorithm is to estimate reflection coefficients, and from these, autoregressive coefficients, so as to best fit a time series data sequence. Full derivation of the algorithm is detailed by Burg in [1]. Translation of this mathematical formulation into sequential code, as well as the sequential to parallel conversioris for the four machines, is described my Sammur and Hagan in [14]. (See also [12] for the sequential program.) To maintain consistency with the Sammur and Hagan study, we obtained the original Burg programs from the researchers, including sequential versions in Fortran and Pascal and parallel versions iri MPP Pascal and iPSC Fortran.

A standard sequential implementation of the Burg algorithm is shown in Fig. 1 [14]. The algorithm recursively estimates the parameters for an $n$-th order system from the known parameters of an $(n-1)$-th order system. The parameters at eacil stage of the recursion are estimated so as to minimize the arithmetic mean of the 
forward and backward prediction errors with respect to $a[n]$, the $n$-th coefficient of the autoregressive model. Specifically, it calculates a specified number of reflection coefficients $(\max )$ given a time series data set $(x)$ having $m$ data points. From the time series, a set of forward $(e)$ and backward $(b)$ prediction errors are determined. The $e$ 's and $b$ 's are recursively updated so the reflection coefficients $(c)$ can be calculated. If desired, the autoregressive coefficients, $a$, can also be calculated. The main step in this algorithm, identified as Loop 1 , is a time shift/inner product operation. It involves a time shift of the $b$ array relative to the e array and incorporates three inner product operations. The step identified as Loop 2 is the updating of the forward and backward prediction errors. It involves vector scale/add operations and statement-level data dependencies. As will be shown in the next section, these two steps, as well as the calculation of $c$, are amenable to parallelization. Loop $I$ incurs communication, and the calculation of $c$ becomes a synchronization barrier between Loop $I$ and Loop 2.

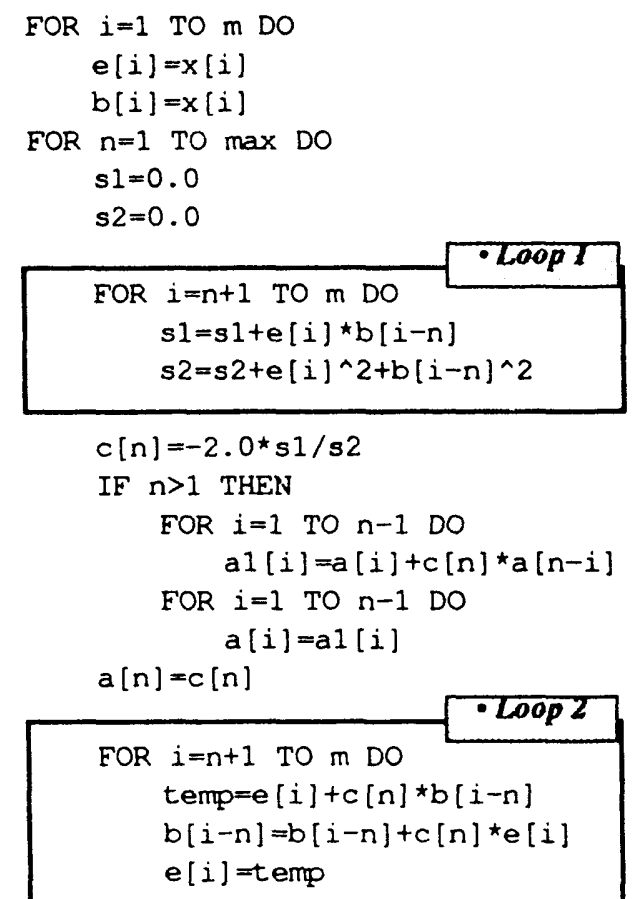

FIG. 1. Sequential Burg algorithm

The operation count for this algorithm in terms of problem size parameters max and $m$, excluding the calculation of the autoregressive cocfficients, is: $(10 \times m \times \max )-\left(5 \times \max ^{2}\right) . m$ is typically much larger than max. While exact values depend on the application, in practice it is common for max to take values between 10 and 50, and for $m$, between 1000 and 10000 [12]; larger $m$ are practical for some applications [14]. Of these operations, Loop 1 accounts for 1.5 times as many operations as Loop 2. Work, measured as the number of floating point operations performed, varies linearly with problem size; storage also varies linearly.

\subsection{Summary of Performance Results}

The Burg algorithm was implemented on four different parallel computers in the earlier study: the Intel iPSC/2, the Denelcor HEP, the NASA/Goodyear MPP, and the Cray X-MP/48. Table I compares execution 
times observed for the parallel versions on the four computers (as reported in [14|) and, as a point of reference, for a serial version on a Sun 4 workstation. Also included in the list are the two parallel machines in this study: the MasPar MP-1 and the nCUBE 2. Each will be discussed in more detail in Section 3. The input problem parameters for this comparison were $\max =10$ and $m=16384$. All machines used single precision (32-bit) floating point arithmetic, which was sufficient for this problem, except the Cray (always double precision).

TABLE I

Burg Execution Times

\begin{tabular}{lc}
\hline Machine & Execution Time (sec.) \\
Sun 4 & 2.85 \\
HEP & 1.68 \\
iPSC/2 & 0.24 \\
MPP & 0.0552 \\
nCUBE 2 & 0.0298 \\
Cray X-MP/48 & 0.0169 \\
MasPar MP-1 & 0.013 \\
\hline
\end{tabular}

Of the four parallel computers in the earlier study, the fastest execution time was delivered by the Cray. Sceing such results was sufficient motivation for us to investigate the performance of the Burg algorithm on our new parallel computers. A result of our efforts is a MasPar execution time that surpasses the mark set by the Cray and that is more than four times faster than its earlier distributed memory counterpart, the MPP. The nCUBE execution time is also impressive; being more than eight times faster than its counterpart, the iPSC, it cstablishes itself as a competitive alternative. The reasons for such notable performance gains are twofold: technology and technique. Relevant changes in machine technology and algorithmic technique are noted in the discussion of each implementation in Section 3.

While revisiting the Sammur and Hagan study and reevaluating Burg algorithm performance, we identified the following assessments (in [14]) which no longer accurately characterize the performance of the algorithm on the distributed memory architectures: (1) The parallelism of the Burg algorithm can be fully exploitcd only if the number of processors is equal to the number of data points which is only feasible if a massively parallel machine like the MPP is used; (2) The calculation of the reflection coefficient at each iteration is a serial bottleneck that limits the algorithm performance (iPSC/2); and (3) The communication overhead incurred from the four-byte message between nodes limits linear speedup to smail numbers of nodes and large nuinbers of data points (iPSC/2). These assessments hold in the context of the earlier study, but that is because they are a direct consequence of the machines and methods being used. For (1), the cross-architectural comparison yielded poor performance on the coarser grained parallel machines because these machines are less scalable than the MPP for executing the Burg program. For (2), the calculation of the reflection coefficient requires a global sum operation for which a costly serial algorithm was used (an efficient parallel algorithm is now well known for hypercubes). And for (3), single-word data transfers can occur in both linear shift and global sum operations, and the transfer time is dominated by the iPSC/2's large message startup overhead. Although such conclusions are indecd informative, care should be taken not to apply them more generally as an evaluation of the mapping of the algorithm onto an architecture. What is being evaluated is the implementation of the algorithm on a machine. Even though our ultimate objective is to correlate performance with classes of architectures and algorithms, it would be a mistake to exclusively let the performance of one machine in an architectural class stand as an indicator of performance for the whole class, thus possibly biasing the use of other machines. This is exemplified in the performance update presented in Section 3. The perfor- 
mance update focuses not on the question, "How fast does the program run?", but on the more clucidating question, "What are the dynamics of program execution?".

\section{PERFORMANCE UPDATE}

The following sections describe our study, in particular the parallel Burg algorithm, its implementation on the MasPar MP-1 and nCUBE 2 computers, and the observed performance on these two machines.

\subsection{General Parallel Implementation}

A generalized parallel version of the Burg algorithm is shown in Fig. 2. The technique of domain decomposition or data parallelism (that is, distributing the data across the processor ensemble) is used. We incorporated the mappings specified in the Sammur and Hagan study. That is, a scattered decomposition [2], as was used for the MPP, is used for the MasPar implementation. And a block decomposition [2], as was uscd for the iPSC, is used for the nCUBE implementation. The concept of a processor "tile" is introduced in this study to accommodate mappings with multiple data points allocated to each processor. A tile is a set of distributed datums, one per processor. Access to a tile cari be done in parallel. Scattered decomposition on MasPar-like machines typically results in large tiles and a small number of datums per processor, that is, a small number of tiles. Conversely, block decomposition on nCUBE-like machines typically results in smaller tiles and a larger number of datums per processor (i.e., number of tiles or block size).

A natural choice is to distribute the one-dimensional $e$ and $b$ arrays so as to parallelize Loop 1 and Loop 2 (sec Fig. 1). To account for the time shift and have the calculations involving $e$ and $b$ local to each processor, communication is required to align the respective elements of $e$ and $b$. This is done via a linear shift operation. Observe that the number of communication steps required for the linear shift of $e$ in Loop $I$ depends on the decomposition (constant for block decomposition and varying linearly with the number of tiles for scattered decomposition). Each lincar shift is accompanied by a zero assignment to $b$ so as to maintain algorithm uniformity and then effectively nullify the superfluous calculations. A second communication phase is required during the calculation of $c$ to accumulate the global sums from the partial sums among all processors. The cost of such an operation depends on the machine, and typically involves a logarithmic to linear number of steps with respect to the machine size; it is independent of the problem size.

\subsection{MasPar Implementation: A SIMD Update}

The MasPar MP-1 is a distributed memory massively parallel SIMD computer [9]. It has a high-speed two-dimensional toroidal mesh topology with eight-way nearest neighbor connections (the Xnet) and a router handling non-neighbor communications among subsets of processors. The Data farallel Unit (DPU) consists of an A rray Control Unit (ACU) and a Processing Element (PE) Array. The ACU operates at $12.5 \mathrm{MHz}$. Each $\mathrm{PE}$ is a custom load/store 4-bit processor with registers, floating-point hardware, and $16 \mathrm{KBytes}$ of memory. The machine in the SCL currently has 8192 PEs arranged in a $128 \times 64$ array (which is the actual interconnection network supported by the Xnet). Two languages are supported: MPL, an extension of C, with explicit plural data constructs and interprocessor communication primitives; and MPFortran, an implementation of Fortran90.

The execution time reported in Table $I$ is for an MPL version of the Burg algorithm using single-precision floating-point arithmetic. Phases of the parallel algorithm coincide with those in Fig. 2. A one-dimensional array of size $m$ is distributed across the PEs, and a linear PE array is simulated (via a snakelike method as was done in the MPP study). The linear shift operation is implemented by a combination of two Xnet operations: a send to the north by Column 0's PEs, and a send to the west by all PEs. These are done for each tile in turn, 


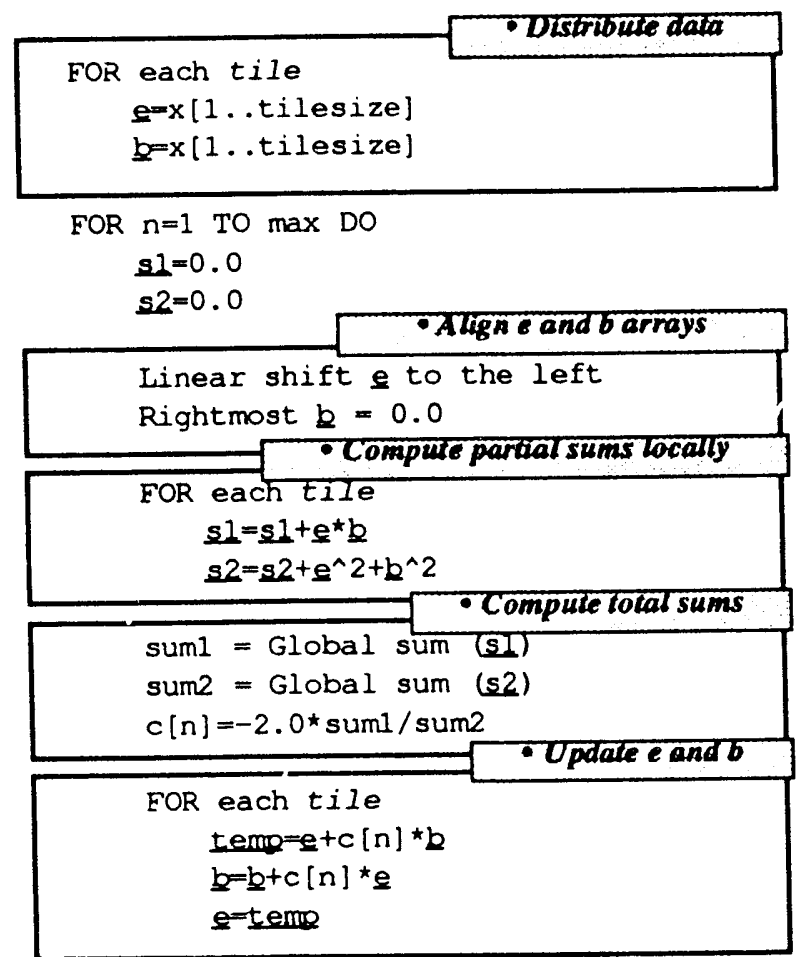

Notation: distributed variable

FIG. 2. Parallel Burg algorithm

with a tinal shift between tiles on the last PE only. Each global sum, enroute to calculating a coefficient, is obtained from an intrinsic ReduceAdd function applied to the partial sums. Fig. 3 is a timing profile illustrating the phases of execution $(a-h)$ and processor activity (grayscale) over time for selected PEs (here, a slice of sixteen PEs in Row 0 and the last PE). The slice of time spans the iteration for the first reflection coefficent for a run with $m=8192$ data points (i.e., one "tile" or one data point per processor). Idle time occurs when processors are disabled (removed from the "active set"). For example, during phase $b$ only Column 0 PEs are active, as shown. Note also the longer time interval for phase $b$ versus phase $c$, despite both being Xnet operations. This is because phase $b$ incurs the overhead of disabling PEs. The Xnet is a resource to be utilized, especially in light of the fact that doing a single-precision multiply is two to three times more expensive than sending the operands to an adjacent PE and receiving the product [2]. Registers should also be utilized because small clusters of PEs are configured to access the same read/write memory unit; significantly better execution times are observed when plural data are explicitly declared as register types.

The scalability of the algorithm is illustrated in Fig. 4, which shows four timing profiles (cf. Fig. 3) for PE 0 corresponding to runs with $m=8192,16384,32768$, and 262144 data points, respectively. That is, runs having $1,2,4$, and 32 tiles, respectively. (Though data sets as large as 32 tiles are not typical in current applications using the Burg algorithm, they are interesting from a performance perspective.) The execution times of phases $a, e$, and, most notably, $g$ are independent of problcm size; times for the other phases increase linearly with the number of tiles. Phase $g$ time depends on machine size, requiring a number of communication steps 


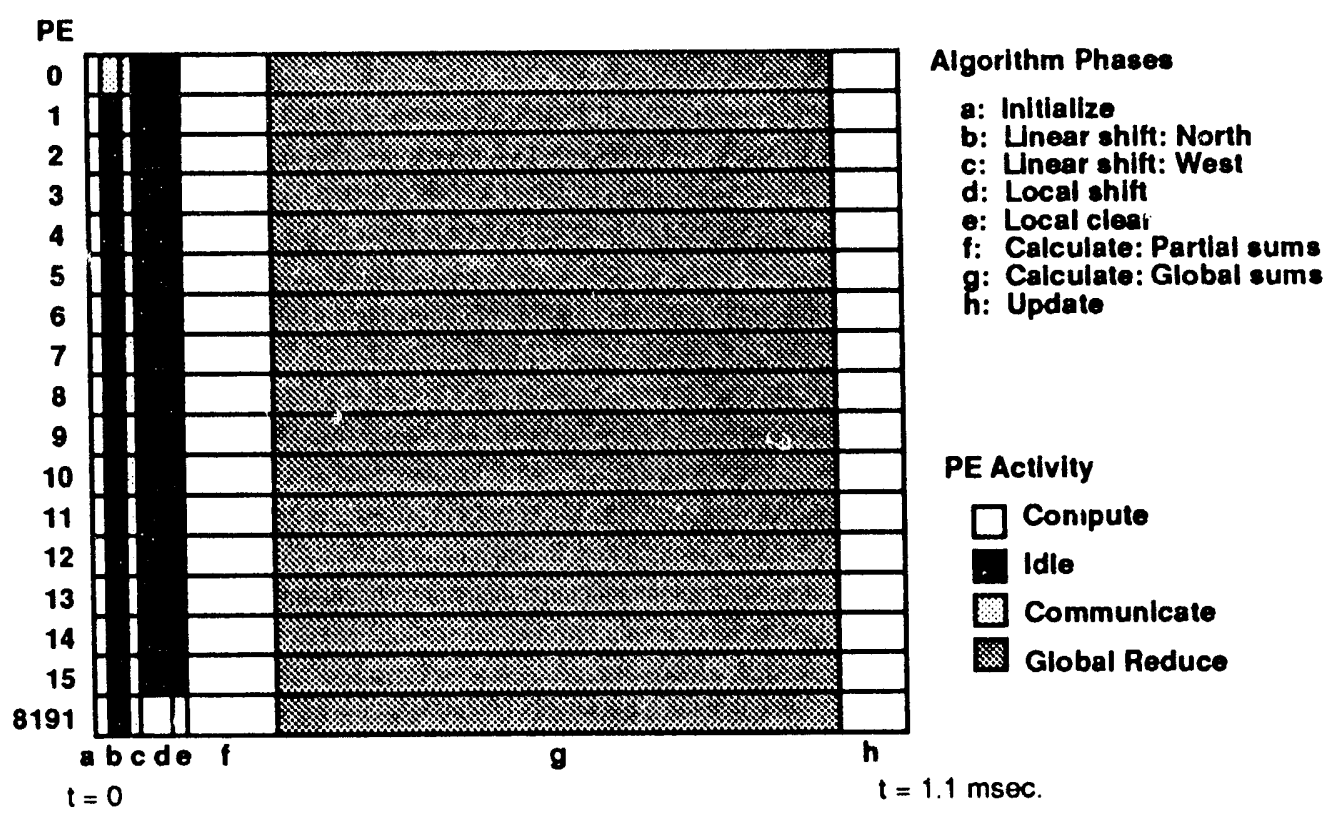

FIG. 3. Execution profile for selected PEs of the MasPar (1 tile)

equal to $\log _{2}$ the numiurer of PEs. Observe that PE utilization is high in each case, even though the global sum operations in phase $g$ are relatively expensive for smaller problem sizes. The performance gain over the MPP has come largely from advanced technological and architectural features of the MasPar. Only minimal improvement can be attributed to algorithmic changes; e.g., tiling was introduced, and this required an optimal ordering of plural control constructs to reduce the overhead of disabling PEs.

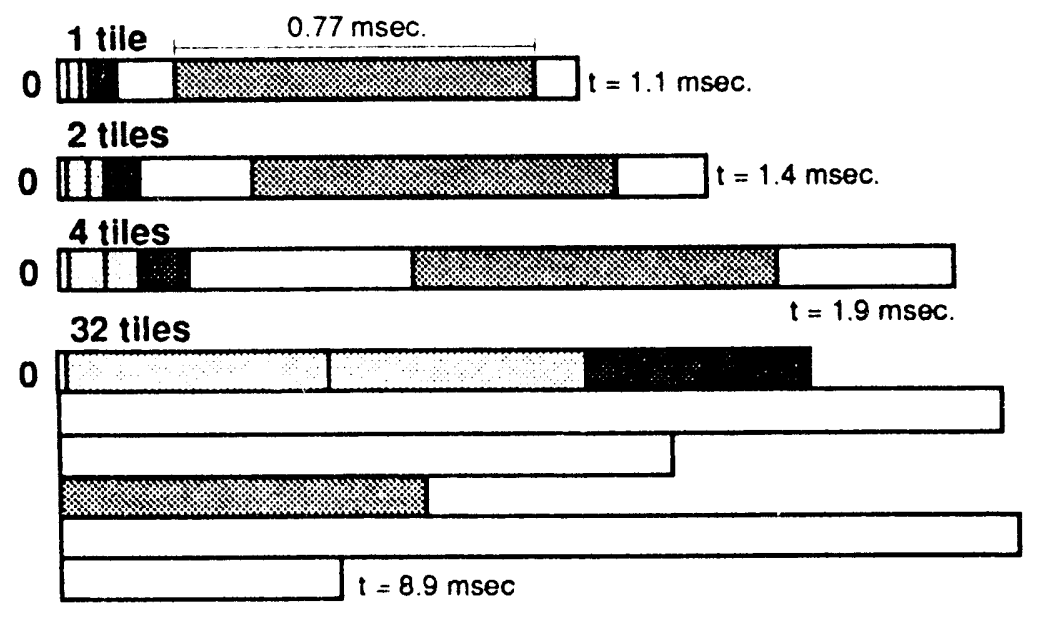

FIG. 4. Execution profile for PE 0 of the MasPar for increasing problem sizes

The exccution profiles, gencrated via program instrumentation and performance visualization [8], provide detailed information about the dynamics of the program executing on the machine. Complementing the graph- 
ical representations of performance are a variety oi statistics and other metrics that help to quantify the observations. Hockney and Jesshope [6] define two useful parameters that provide a quantitative means to individually characterize and collectively compare the performance of parallel (and other) computers for a given program: (1) the maximum or asymptotic performance $r_{\infty}$ - the maximum rate of execution in floating-point operations performed per second (commonly stated as millions of FLOPS, or MFLOPS); and (2) the half-performance length $n_{1 / 2}$ - the number of datums required to achieve half of the maximum performance. Both are experimentally measured quantities obtained by varying the number of datums (problem size, $n$ ) and timing program execution on a machine of a specific ensemble size. $r_{\infty}$ reflects both the peak speed of the machine and the goodness of fit between program and machine. It is calculable from the ratio of expressions: operation count $(n) /$ time $(n)$, as $n$ approaches infinity. The expression in the numerator is determined via program analysis; and, in the denominator, via curve fitting the measured data. $n_{1 / 2}$ can be interpreted as a measurs , of the amount of parallelism in the system, specifically, in the program-machine mapping which produced $r_{\infty}$. It zan be argued that a computer only solves efficiently those problems having $n$ greater than $n_{1 / 2}[6]$. Thus, there is a tradeoff: the higher the value of $n_{l / 2}$, the greater the parallelism, but the more limited is the range of problems that can be solved efficiently.

We can apply these parameters to $($ aracterize the performance of the Burg algorithm on the MasPar MP-1 (and the nCUBE $\lambda, t)$ be discussed in the $x$ xt section). Execution speed ( $r$ ) versus time is shown in Fig. 5 for an 8192-PE system; is. dashed line above the MasPar curve denotes maximum performance, $r_{\infty} \cong 325$ MFLOOPS. Efficiency, defined here as the ratio of actual speed to peak speed $\left(r / r_{\infty}\right)$, is shown in Fig. 6; the dashed line indicates $50 \%$ efficiency, or half of the maximum performance, which corresponds to $n_{1 / 2} \cong 30600$ data points. Observe that there is good "acceleration" in approaching maximum performance; that $n_{1 / 2}$ indicates a high degree of parallelism; and that reasonable efficiencies can be obtained for a range of values of $n$ used for the program. Time is used as the dependent variable (instead of $n$ ) in Figs. 5 and 6 to represent work done by each machine. This is because a fixed-time model of performance measurement has provided "simple" and consistent explanations of performance phenomena we have observed.

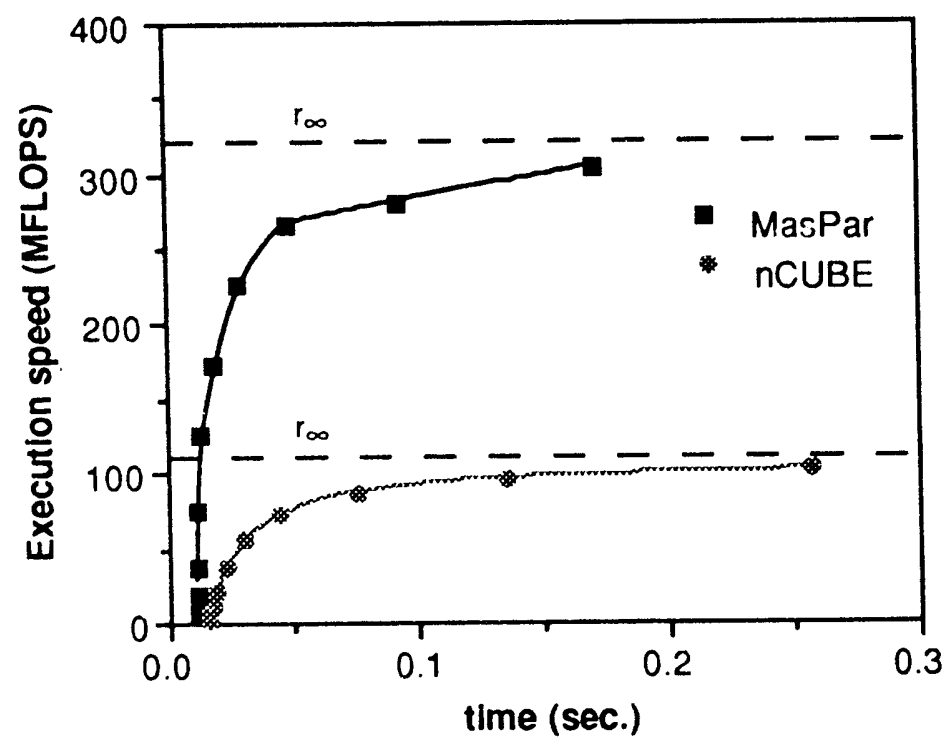

FIG. 5. Execution speed on the MasPar and nCUBE as a function of exccution time 


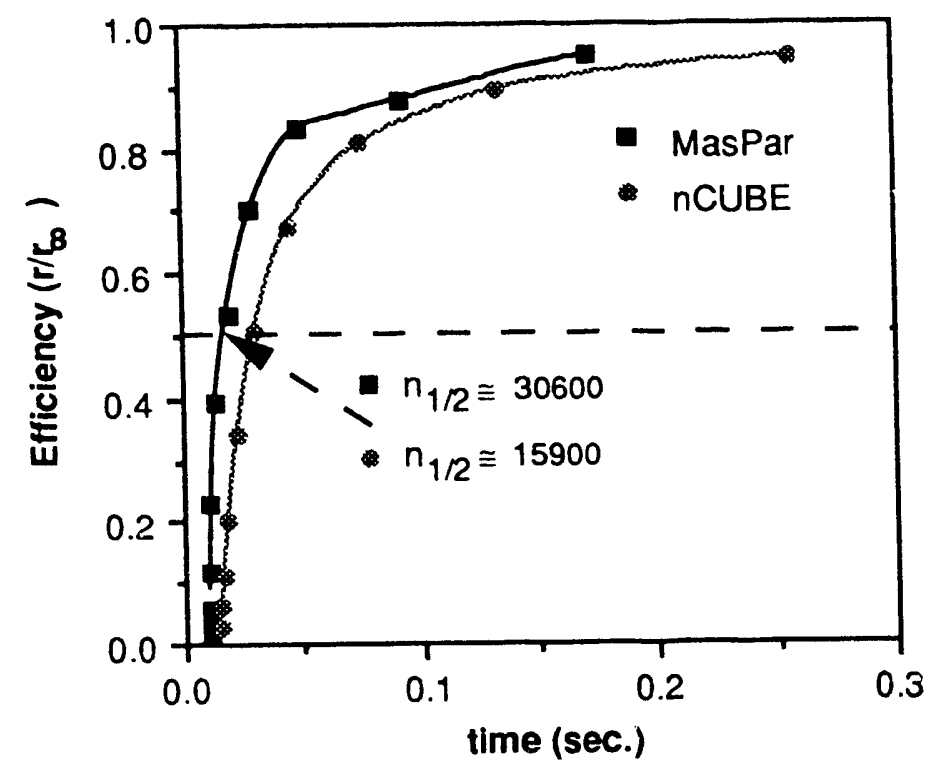

FIG. 6. Exccution efficiency on the MasPar and nCUBE as a function of execution time

\section{3. nCUBE Implementation: A MIMD Update}

The nCUBE 2 is a distributed memory message-passing MIMD computer [10]. It has a hypercube interconnection topology with cut-through routing to non-neighboring processor nodes. Each node is a custom 32bit CISC (Complex Instruction Sct Computer) processor operating at $20 \mathrm{MHz}$. with floating-point hardware, DMA-based bidirectional interprocessor communication channels, and 1 MByte of memory. Standard operating system message-passing procedures incur a $60 \mu \mathrm{sec}$. delay per message transmission due to software overhead. Communication channel bandwidth is $2.2 \mathrm{MBytes} / \mathrm{sec}$. Single-word, near-neighbor messages typically require 70-80 $\mu \mathrm{sec}$. for total transmit time (with no contention). This is a factor of five to ten faster than earlier machines such as the nCUBE 1 and the iPSC/2. Indeed, the lower message latency is one reason that the nCUBE 2 gives a more scalable solution to the Burg algorithm than the iPSC/2 (at 2.8 MBytes/sec. bandwidth and $\cong 500 \mu \mathrm{sec}$. overhead). Though not used yet in this study, new operating system message-passing procedures are now becoming available on the nCUBE that obviate some of the overhead in well-structured cases (for which the Burg algorithm would qualify). The machine in the SCL currently has 64 nodes (a six-dimensional hypercube). Two languages are, supported, $\mathrm{C}$ and Fortran, each with similar features.

The exccution time reported ir. Table $\mathrm{I}$ is for a $\mathrm{C}$ version of the Burg algorithm using single-precision floating-pnint arithmetic and assembler routines from a vector library [4]. Phases of the algorithm coincide with those in Fig. 2. The linear shift operation involves local and global operations: a local shift on the block of array elements "owned" by a processor, and one global linear shift to move elements from processor $i$ to processor $i-1$ (from the start of a block to the end of the preceding block), where the $i$ 's correspond to a onedimensional ordering of the processors. These operations can be partially overlapped using the sequence: write to "left" neighbor, perform local shift, and read from "right" neighbor. This allows the computation to effectively absorb any excess communication delay. Each global sum operation is implemented with an efficient algorithm that utilizes the hypercube interconnect to its full potential. Pairs of nodes exchange partial sums across each hypercube dimension in turn, requiring a number of communication steps equal to the dimension of the hypercube ( $\log _{2}$ of the number of processors). This logarithmic communication cost is in 
stark contrast to the linear cost incurred by the uploading-downloading technique (between Node 0 and all other nodes) used for the iPSC 2 in the earlier study [14]. The global sum is parallel, not serial, in nature and is scalable. These algorithmic changes, primarily to minimize communication time, are the main reason for the greater scalability of the nCUBE solution to the Burg algorithm.

Performance visualization for the nCUBE was done using a combination of tools developed by researchers at Oak Ridge National Laboratory: PICL (Portable Instrumented Communication Library) [3] and ParaGraph [5]. Both are available through netlib. PICL produces an execution trace during an actual run of an instrumented program, and ParaGraph replays this trace using multiple views. Though the current version is limited to displaying a small number of processors, a new version of ParaGraph to be released will accommodate large numbers of processors; scalable views are being developed at the SCL for integration into tools like ParaGraph [13]. One view selected to illustrate the execution of the Burg algorithm is the Feynman Diagram. The Feyn man Diagram is patterned after the diagrams that are used in physics to depict the interactions between particles (see Figs. 7 and 8). Processor activity is indicated by horizontal lines, one for each processor, while messages between processors are depicted by slanted lines between the sending and receiving processor activity lines, indicating the times at which each message was sent and received. A blank horizontal line indicates that a processor is idle waiting to receive a message. Fig. 7 shows a Feynman Diagram for the execution of the Burg algorithm on a 16 node subcube, spanning the iteration for the first reflection coefficient for a run with a block size of 16. Fig. 8 is similar, except with a block size of 256 . In each view, a Gray-code ordering of the nodes was selected for the vertical axis. The first set of messages comprise the linear shift operation. The next four sets of messages constitute the pair-wise exchanges across consecutive dimensions of the hypercube. Observe the uniformity and synchroneity of the communication patterns; observe that no processors ever wait to receive a message. As seen for the MasPar, smaller problem sizes tend to be relatively expensive. These visual depictions of system activity reveal patterns and anomalies from which performance characteristics become perceptible to the user. Critical paths, for example, would suggest a need to redesign the algorithm (which we did).

FEYNMAN DIAGRAM

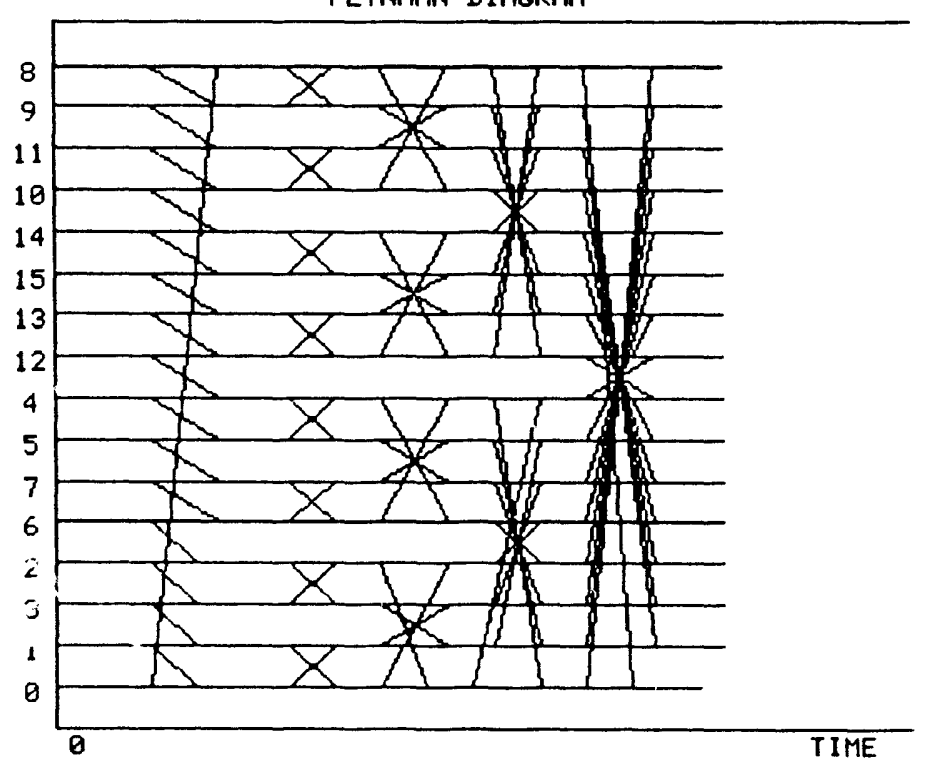

FIG. 7. Execution profile for a 16-node subcube of the nCUBE (block size $=16$ ) 
FEYNMAN DIAGRAM

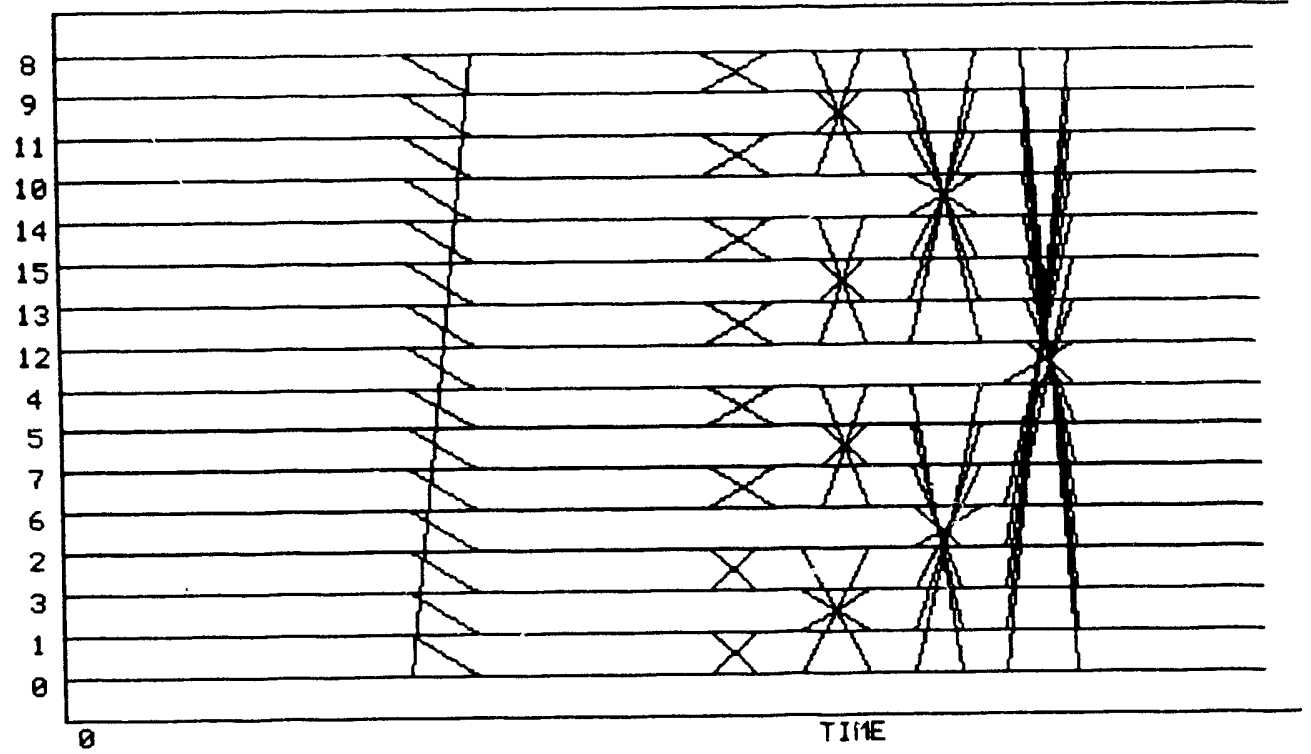

FIG. 8. Execution profile for a 16-node subcube of the nCUBE (block size $=256$ )

As was done in the preceding section for the MasPar, we can apply the $\left(r_{\infty}, n_{1 / 2}\right)$ parameters to characterize quantitatively the performance of the Burg algorithm on the nCUBE 2. Execution speed $(r)$ versus time is shown in Fig. 5 for a 64 -node system; the dashed line above the nCUBE curve denotes maximum performance, $r_{\infty} \cong 110$ MFLOPS. Efficiency $\left(r / r_{\infty}\right)$ is shown in Fig. 6 (also for 64 nodes); the dashed line indicates $50 \%$ efficiency, or half of the maximum performance, which corresponds to $n_{1 / 2} \cong 15900$ data points. By comparing the MasPar and nCUBE performance curves, we see that, although the MasPar has a higher maximum performance than the nCUBE, they have similar efficiencies. The factor of two difference in $n_{1 / 2}$ correctly reveals that the MasPar is a more highly parallel machine, but it also suggests that the nCUBE's performance characteristics for the Burg algorithm are not too unlike the MasPar's. Differences in maximum performance can be traced in part to the rated peak speed of the machines: 556 single-precision MFLOPS for the MasPar, and 171 for the nCUBE.

A final comparison between the two machines is shown in Fig. 9. A timing profile (cf. Fig. 3) for processor 0 on each machine is displayed for the iteration of the first reflection coefficient and for problem sizes that yield about equal execution time ( $m=4096$ data points, or 128 per each of 32 nodes, on the nCUBE; and $m=$ 8192 , or 1 per each of 8192 PEs, on the MasPar). Observe the analogous behavior on the two machines despite the contrasting machine granularities.

\section{DISCUSSION}

In the process of reevaluating Burg algorithm performance, we found that the algorithm maps efficiently to small-scale as well as large-scale computers, including both SIMD and MIMD distributed memory systems. Our study was done in the context of a massively-parallel SIMD computer, the 8192-PE MasPar, and a modestly-parallel MIMD computer, the 64-node nCUBE. Although the performance interplay between machine 


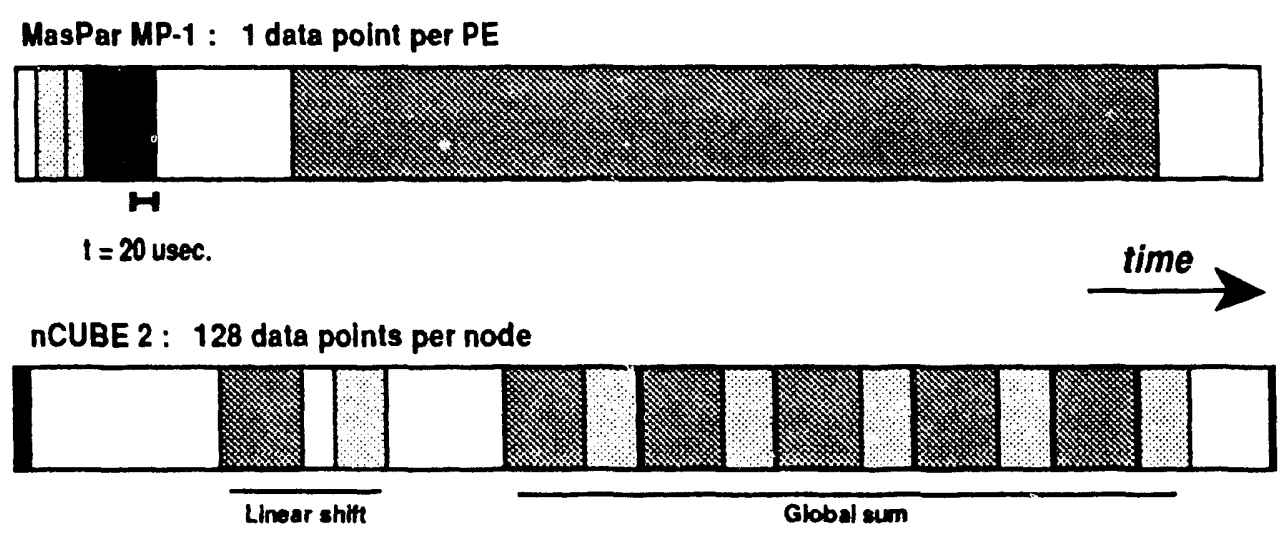

FIG. 9. Comparison of execution profiles for processor 0 on the nCUBE and MasPar

ensemble size and problem size results in lower efticiencies for smaller problem sizes on both the MasPar and the nCUBE, the algorithm does exhibit a high degree of parallelism and scalability on both computers.

Regarding the assessments stated in Section 2, we make the following statements. (1) The parallelism of the Burg algorithm was exploited on both the MasPar and the nCUBE, and in each case, optimal performance was observed when the number of data points exceeded the number of processors. (2) The calculation of the reflection coefficient, in particular the global sum operation, is a parallel operation on both architectures, involving highly uniform and efficient communication. (3) Commuication overhead on hypercube computers has been (and continues to be) reduced so that short messages are not as costly as they once were. For the algorithm on the nCUBE, communication is structured, both as a natural consequence of the algorithm and by explicit programming, so that little (if any) time is spent waiting for messages. As future machines impose shorter message transmission times, the result will be finer grained machines, making the algorithm-machine combination more scalable. Thus, efficiencies, especially for smaller problem sizes, will improve.

In conclusion, parallel supercomputers having a distributed memory architecture are suitable for signal processing algorithms similar to the Burg algorithm and are competitive altenatives to the more conventional vector supercomputers. Scalability remains a hard to define parallel processing concept, one that has and will result in changing perceptions of performance as it becomes better understood and applied. Finally, the utility of appropriate performance metrics and performance instrumentation and visualization to interpret the complex operation of large-scale systems cannot be overstated.

\section{ACKNOWLEDGMENTS}

The authors especially thank Gretchen Vogel, Susan Helfter, and Michael Carter for their technical assistance throughout the study. Thanks are also extended to Drs. Martin Hagan and Nidal Sammur of Oklahoma State University for their cooperation in acquiring the code from their study. This project was funded in part by the Iowa State University Women in Science and Engineering Program. The work was performed at the Scalable Computing Laboratory which is funded by lowa State University and the Ames Laboratory-USDOE. 


\section{REFERENCES}

1. Burg, J. P. Maximum entropy spectral analysis. In Modern Spectrum Analysis, edited by Childers. New York: IEEE Press, 1978.

2. Fox, G., Johnson, M., Lyzenga, G., Otto, S., Salmon, J., and Walker, D. Solving Problems on Concurrent Processors. Englewood Cliffs: Prentice-Hall, 1988.

3. Geist, G., Heath, M., Peyton, B., and Worley, P. A machine-independent communication library. Proceedings of the Fourth Conference on Hypercubes, Concurrent Computers, and Applications. Los Altos, CA: Golden Gate Enterprises, 1990.

4. nCUBE 2 Math Library Manual. nCUBE Corp., 1990.

5. Heath, M. T. Visual animation of parallel algorithms for matrix computations. Proceedings of the Fifth Distributed Memory Computing Conference. New York: IEEE Computer Society, 1990.

6. Hockney, R. W. and Jesshope, C. R. Parallel Computers 2: Architecture, Programming, and Applications. Bristol: IOP Publishing Ltd., 1988.

7. Jangi, S. and Jain, Y. Embedding spectral analysis in equipment. IEEE Spectrum Vol. 28, No. 2 (Feb., 1991).

8. Koskela, R. and Simmons, M., editors. Performance Instrumentation and Visualization. New York: ACM \& Addison-Wesley, 1990.

9. MasPar MP-1 Standard Programming Manuals. MasPar Computer Corp., 1990.

10. nCUBE 2 Programmer Reference Manual and Processor Manual. nCUBE Corp., 1990.

11. Nussbaum, D. and Agarwal, A. Scalability of parallel machines. Communications of the ACM Vol. 34, No. 3 (March, 1991).

12. Press, W., Flannery, B., Teukolsky, S., and Vetterling, W. Numerical Recipes: The Art of Scientific Computing. Cambridge: Cambridge University Press, 1989.

13. Rover, D. T. and Wright, C. T. Pictures of performance: highlighting program activity in time and space. Proceedings of the Fifth Distributed Memory Computing Conference. New York: IEEE Computer Society, 1990.

14. Sammur, N. M. and Hagan, M. T. Mapping signal processing algorithms on parallel architectures. Journal of Parallel and Distributed Computing Vol. 8, No. 2 (Feb., 1990).

DIANE ROVER received the B.S. degree in computer science in 1984, the M.S. degree in computer engineering in 1986 , and the Ph.D. degree in computer engineering in 1989, all from Iowa State University. From 1985 to 1988, she was awarded an IBM Graduate Fellowship. In 1986, Dr. Rover was an intern with McDonnell Douglas Corporation, and in 1987, with the IBM Thomas J. Watson Research Center. Since 1983, she has been a Technical Education Consultant for IBM. She is currently a postdoctoral researcher in the Scalable Computing Laboratory at the Ames Laboratory. Her research interests include parallcl processing, computer architecture, performance evaluation, instrumentation, and performance visualization. Dr. Rover is a member of the IEEE Computer Society, the Association for Computing Machinery, Sigma Xi, and the Society of Women Engineers.

VICKI TSAI is a senior at Ames High School, Ames, Iowa. She is a National Merit Scholarship finalist. During Summer, 1990, she was an intern in the Scalable Computing Laboratory at the Ames Laboratory as part of the lowa State University Women in Science and Engincering Summer Internship Program. She is currently a research helper in the SCL.

YIN-SHAN CHOW is an undergraduate student in Electrical Engineering at lowa State University. During Summer, 1990, she was an intern in the Scalable Computing Laboratory at the Ames Laboratory as part of the Iowa State University Women in Science and Engineering Summer Internship Program. She is currently a research helper in the SCL. Her 
interests are in the areas of parallel computing, opto-electronics, and analog and digital design. She is a member of the IEEE, Eta Kappa Nu, and Tau Beta Pi.

JOHN GUSTAFSON received the B.S. degree in applied mathematics from Caltech (1977), the M.S. and the Ph.D. at Iowa State University in 1981 and 1982, respectively. He was Product Development Manager and Senior Staff Scientist at Floating Point Systems from 1982 to 1986, Staff Scientist at nCUBE from 1986 to 1987, and a Member of the Technical Staff at Sandia National Laboratories from 1987 to 1989. His work on the 1024-processor hypercube at Sandia, with colleagues Gary Montry and Robert Benner, won the inaugural Gordon Bell award in 1988. Since 1989, he has led research efforts in massively-parallel computing at the Ames Laboratory. Dr. Gustafson is a Subject Area Editor for Performance Evaluation for the Journal of Parallel and Distributed Computing. His interests include computational physics and chemistry, novel performance metrics, and parallel algorithms. He is a member of SIAM. 


\section{DISTRIBUTION LIST}

Ames Laboratory

Document Library

Iowa State University

201 Spedding Hall

Ames, Iowa 50011-3020

(6)

Chicago Operations

Patent Counsel

9800 South Cass Avenue

Argonne, Illinois 60439

Mr. Daniel Griffen

ISURF

315 Beardshear Hall

(1)

Technical Information Center

U.S. Department of Energy

P.O. Box 62

Oak Ridge, Tennessee 37830

Scalable Computing Laboratory 236 Wilhclm Hall

Ames Laboratory

Dr. Diane Rover

Dept. of Electrical Engineering Michigan State University

East Lansing, Michigan 48824 

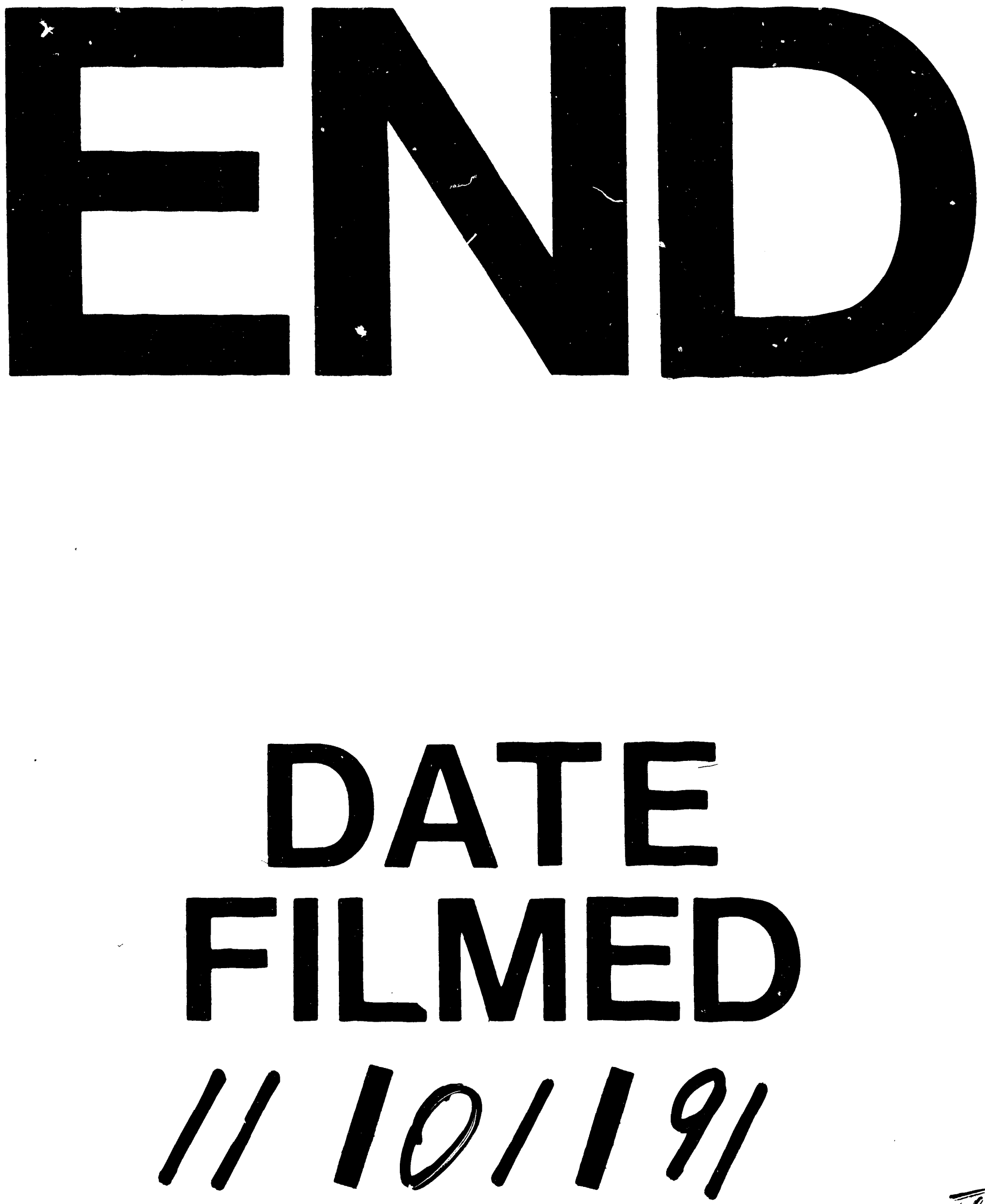

$\overline{1}$ 
